\title{
10 NEW VARIABLE STARS DETECTED BY CRTS
}

\author{
Andrey Galinskiy, Sergei Dubrouski, Igor Baluk \\ The Regional Centre of Technical Creativity of Children and Youth (RCTCCY), \\ Oktyabrya avenue, 36 A, Gomel, Belarus \\ andigall@yandex.ru,toliman@tut.by,balig@tut.by
}

ABSTRACT. 10 new variable stars were found by CRTS.

Key words: Stars: new variable stars.

The authors carried out investigation using the time series photometry data from the Catalina Real-Time Transient Survey (CRTS) in 2013-2015. About 3,000 new variable stars were found. The data for 10 the most recently discovered stars are presented in the journal Odessa Astronomical Publications.

\section{SSS J041936.0-502614}

The star was discovered by I.I.Baluk.

The UCAC4 position of the star: RA $=04^{\mathrm{h}} 19^{\mathrm{m}} 35.937^{\mathrm{s}}$, Dec $=-50^{\circ} 26^{\prime} 51^{\prime \prime} .03$.

The star's catalogue identifiers: the 2MASS identifier J04193593-5026149; the USNO identifier - B1.0 03950035953; the UCAC4 identifier - 198-004300.

The star's variability type: EA, the peak brightness is $15.40^{\mathrm{m}}$; the minimum brightness is $16.07^{\mathrm{m}}$ (in the $\mathrm{C}$ and $\mathrm{V}$ band); the secondary minimum brightness is $15.70^{\mathrm{m}}$ (in the $\mathrm{C}$ and $\mathrm{V}$ band). The star's light ephemerides are plotted in Figure 1. $\mathrm{D}=0.18$.

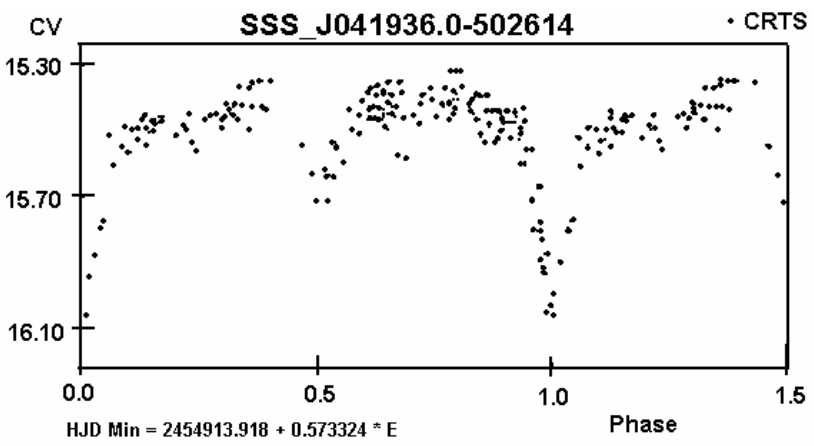

Figure 1

\section{SSS_J065711.3-512110}

The star was discovered by I.I.Baluk.

The UCAC4 position of the star: RA $=06^{\mathrm{h}} 57^{\mathrm{m}} 11.314^{\mathrm{s}}$, Dec $=-51^{\circ} 21^{\prime} 10^{\prime \prime} .47$.
The star's catalogue identifiers: the 2MASS identifier J06571131-5121104; the USNO identifier - B1.0 03860064392; the UCAC4 identifier - 194-009935.

The star's variability type: SRA, the peak brightness is $11.80^{\mathrm{m}}$; the minimum brightness is $12.70^{\mathrm{m}}$ (in the $\mathrm{C}$ and $\mathrm{V}$ band). The star's light ephemerides are plotted in Figure 2. $\mathrm{J}-\mathrm{K}=1.35$.

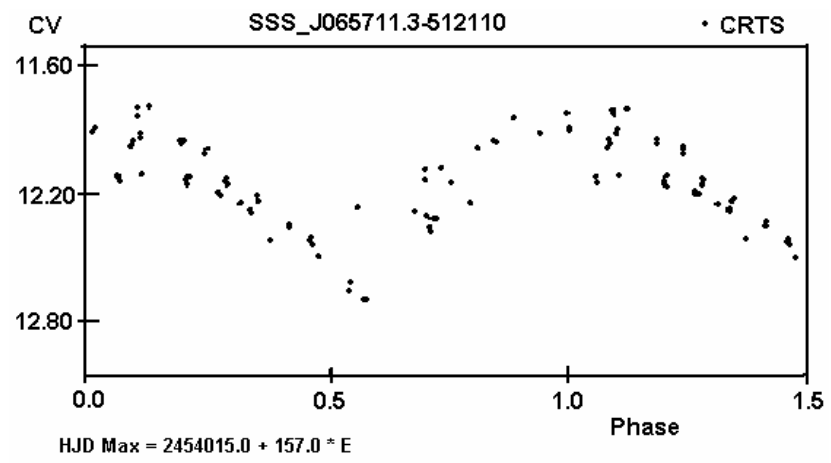

Figure 2

\section{SSS_J195851.4-521601}

The star was discovered by I.I.Baluk.

The PPMXL position of the star: RA $=19^{\mathrm{h}} 58^{\mathrm{m}} 51.384^{\mathrm{s}}$, Dec $=-52^{\circ} 16^{\prime} 01^{\prime \prime} .80$.

The star's catalogue identifiers: the 2MASS identifier J19585138-5216017; the USNO identifier - B1.0 03771050512.

The star's variability type: EW, the peak brightness is $17.10^{\mathrm{m}}$; the minimum brightness is $17.40^{\mathrm{m}}$ (in the $\mathrm{C}$ and $\mathrm{V}$ band).

The star's light ephemerides are plotted in Figure 3.

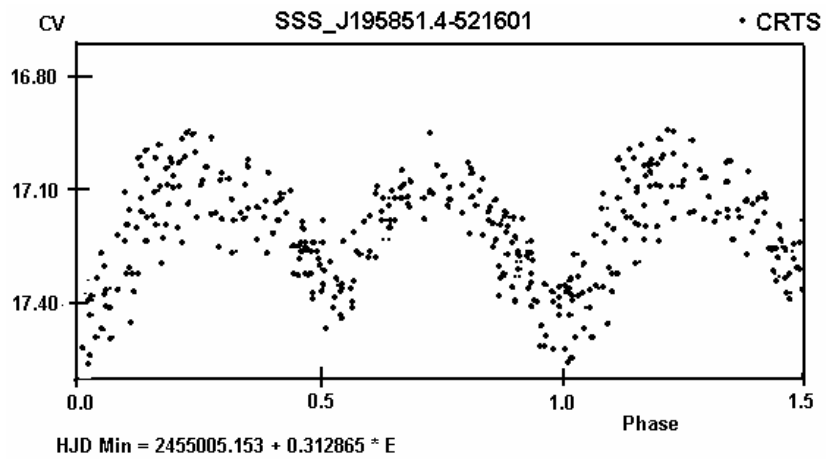

Figure 3 


\section{SSS_J020922.2-525228}

The star was discovered by A.L.Galinskiy.

The UCAC4 position of the star: RA $=02^{\mathrm{h}} 09^{\mathrm{m}} 22.139^{\mathrm{s}}$, Dec $=-52^{\circ} 52^{\prime} 28^{\prime \prime} .37$.

The star's catalogue identifiers: the 2MASS identifier J02092213-5252284; the USNO identifier - B1.0 03710017475; the GSC identifier - 08483-00062; the UCAC4 identifier - 186-001925.

The star's variability type: SR, the peak brightness is $14.90^{\mathrm{m}}$; the minimum brightness is $15.29^{\mathrm{m}}$ (in the C and V band). The star's light ephemerides are plotted in Figure 4. $\mathrm{J}-\mathrm{K}=1.18$.

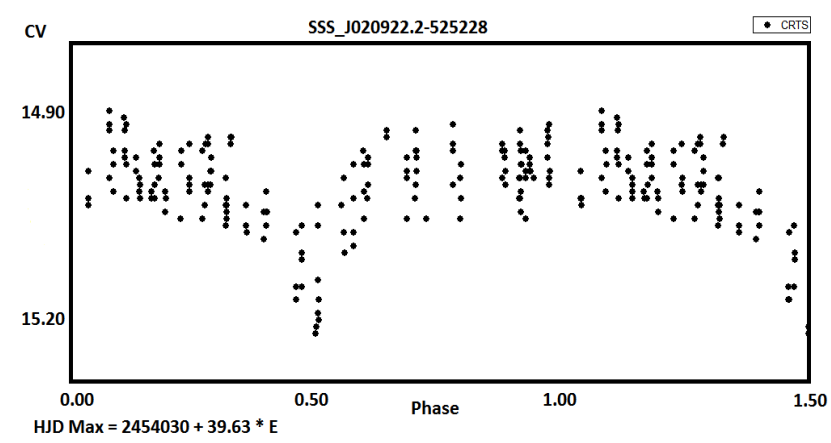

Figure 4

\section{SSS_J035759.1-515819}

The star was discovered by A.L.Galinskiy.

The PPMXL position of the star: RA $=03^{\mathrm{h}} 57^{\mathrm{m}} 59.139^{\mathrm{s}}$, Dec $=-51^{\circ} 58^{\prime} 19^{\prime \prime} .39$.

The star's catalogue identifiers: the 2MASS identifier J03575913-5158193; the USNO identifier - B1.0 03800047211; the GSC identifier - 08073-00336.

The star's variability type: SR, the peak brightness is $15.50^{\mathrm{m}}$; the minimum brightness is $15.98^{\mathrm{m}}$ (in the $\mathrm{C}$ and $\mathrm{V}$ band). The star's light ephemerides are plotted in Figure 5. $\mathrm{J}-\mathrm{K}=1.21$.

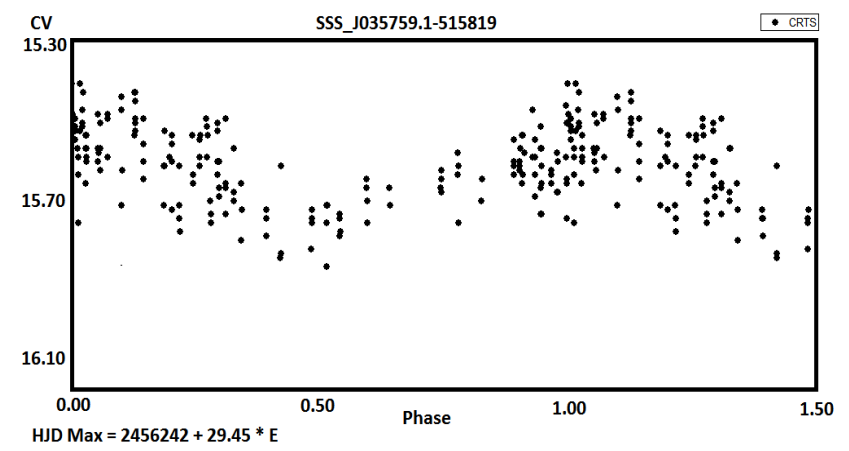

Figure 5

\section{SSS_J073103.3-525443}

The star was discovered by A.L.Galinskiy.

The UCAC4 position of the star: RA $=07^{\mathrm{h}} 31^{\mathrm{m}} 03.297^{\mathrm{s}}$, $\operatorname{Dec}=-52^{\circ} 54^{\prime} 43^{\prime \prime} .63$.
The star's catalogue identifiers: the 2MASS identifier J07310329-5254436; the USNO identifier - B1.0 03700086299; the UCAC4 identifier - 186-012398.

The star's variability type: SR, the peak brightness is $12.10^{\mathrm{m}}$; the minimum brightness is $12.90^{\mathrm{m}}$ (in the $\mathrm{C}$ and $\mathrm{V}$ band). The star's light ephemerides are plotted in Figure 6. $\mathrm{J}-\mathrm{K}=1.27$.

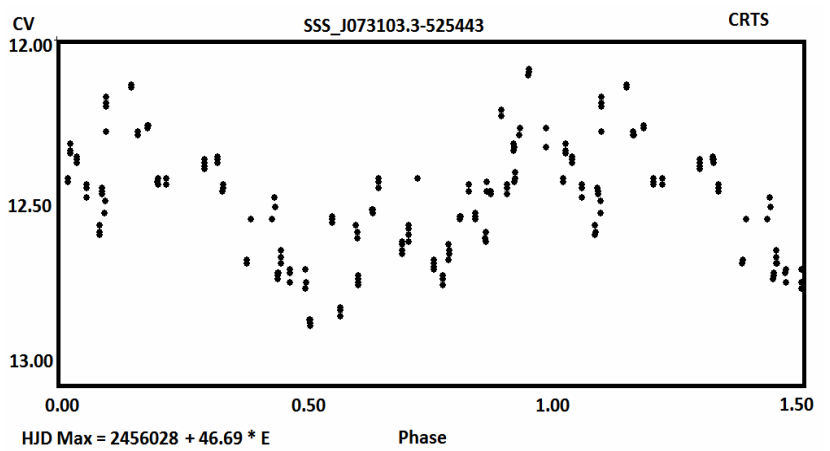

Figure 6

\section{SSS_J100550.3-282525}

The star was discovered by A.L.Galinskiy.

The UCAC4 position of the star: RA $=10^{\mathrm{h}} 05^{\mathrm{m}} 50.317^{\mathrm{s}}$, Dec $=-28^{\circ} 25^{\prime} 25^{\prime \prime} .14$.

The star's catalogue identifiers: the 2MASS identifier J10055031-2825251; the USNO identifier - B1.0 06150234047; the GSC identifier - 06629-01092; the UCAC4 identifier - 308-062711.

The star's variability type: EA, the peak brightness is $14.59^{\mathrm{m}}$; the minimum brightness is $14.90^{\mathrm{m}}$ (in the $\mathrm{C}$ and $\mathrm{V}$ band); the secondary minimum brightness is $14.69^{\mathrm{m}}$ (in the $\mathrm{C}$ and $\mathrm{V}$ band). The star's light ephemerides are plotted in Figure 7. $\mathrm{D}=0.16$.

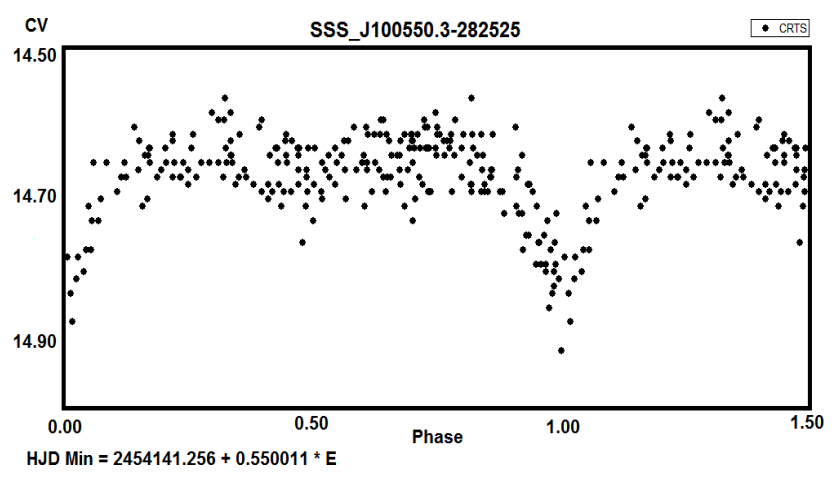

Figure 7

\section{SSS_J101916.8-281924}

The star was discovered by A.L.Galinskiy.

The UCAC4 position of the star: RA $=10^{\mathrm{h}} 19^{\mathrm{m}} 16.838^{\mathrm{s}}$, Dec $=-28^{\circ} 19^{\prime} 25^{\prime \prime} .02$.

The star's catalogue identifiers: the 2MASS identifier J10191683-2819250; the USNO identifier - B1.0 06160240796; the GSC identifier - 06630-00805; the UCAC4 identifier - 309-061750. 
The star's variability type: EB, the peak brightness is $14.30^{\mathrm{m}}$; the minimum brightness is $14.60^{\mathrm{m}}$ (in the $\mathrm{C}$ and $\mathrm{V}$ band); the secondary minimum brightness is $14.45^{\mathrm{m}}$ (in the $\mathrm{C}$ and $\mathrm{V}$ band).

The star's light ephemerides are plotted in Figure 8.

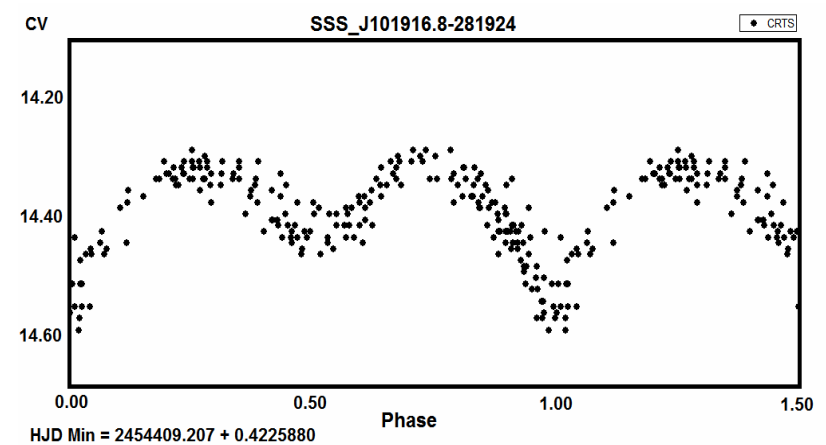

Figure 8

\section{SSS_J073149.9-505012}

The star was discovered by A.L.Galinskiy.

The UCAC4 position of the star: RA $=07^{\mathrm{h}} 31^{\mathrm{m}} 49.879^{\mathrm{s}}$, Dec $=-50^{\circ} 50^{\prime} 12^{\prime \prime} .48$.

The star's catalogue identifiers: the 2MASS identifier J07314986-5050124; the USNO identifier - B1.0 03910077713; the GSC identifier - 08145-00452; the UCAC4 identifier - 196-013096.

The star's variability type: SRA, the peak brightness is $11.00^{\mathrm{m}}$; the minimum brightness is $12.10^{\mathrm{m}}$ (in the C and V band). The star's light ephemerides are plotted in Figure 9. $\mathrm{J}-\mathrm{K}=1.36$.

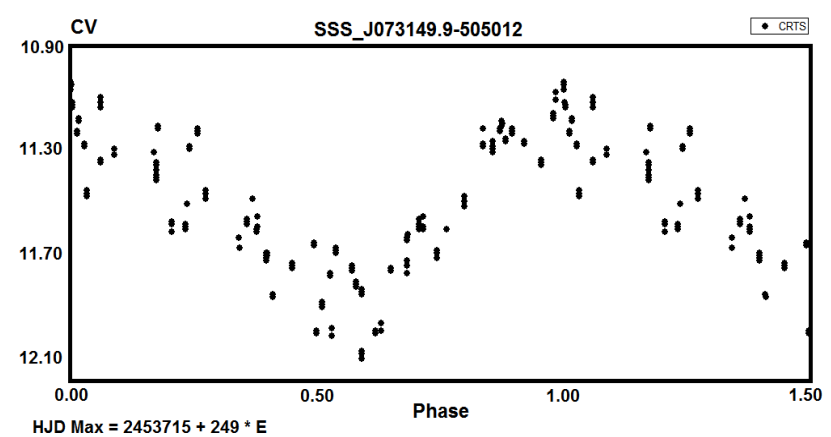

Figure 9

\section{SSS_J200359.4-432015}

The star was discovered by A.L.Galinskiy.

The UCAC4 position of the star: RA $=20^{\mathrm{h}} 03^{\mathrm{m}} 59.430^{\mathrm{s}}$, Dec $=-43^{\circ} 20^{\prime} 15^{\prime \prime} .83$.

The star's catalogue identifiers: the 2MASS identifier J20035942-4320158; the USNO identifier - B1.0 04660733718; the GSC identifier - 07959-00363; the UCAC4 identifier - 234-176574.

The star's variability type: EB, the peak brightness is $13.85^{\mathrm{m}}$; the minimum brightness is $14.05^{\mathrm{m}}$; the secondary minimum brightness is $13.95^{\mathrm{m}}$ (in the $\mathrm{C}$ and $\mathrm{V}$ band).

The star's light ephemerides are plotted in Figure 10.

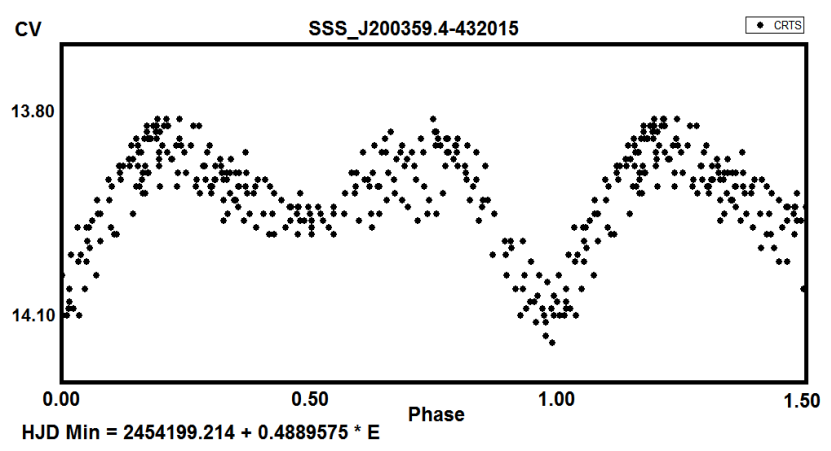

Figure 10

Acknowledgements. The authors are grateful to Ivan S. Bryukhanov, Sergey M. Andrievsky, and Alexander Pobiacha for their kind support and assistance.

\section{References}

Catalina Real-time Transient Survey (A.J.Drake et al. 2009, ApJ, 696, 870).

Lafler J., Kinman T. D.: 1965, Ap. J. Suppl., 11, 216. 